\title{
ON MALLIAVIN'S COUNTEREXAMPLE TO SPECTRAL SYNTHESIS
}

\author{
BY IAN RICHARDS ${ }^{1}$
}

Communicated by N. Levinson, March 11, 1966

Malliavin's disproof of spectral synthesis breaks into two main parts: the first uses a certain "operational calculus," while the second involves a construction. Here we will be concerned only with the second part. The required construction is complicated, and several versions of it have been given [2], [3], [5]. In this note we describe an approach which appears to be somewhat simpler.

It should be mentioned that Varopoulos [6], [7] has recently given a completely different disproof of spectral synthesis, using tensor products of Banach algebras. However Malliavin's original counterexample, although difficult to construct, gives a very powerful result: for instance it shows that spectral synthesis fails even for principal ideals.

(Malliavin's results imply the existence of an $f \in A(\Gamma)$ such that $f, f^{2}, f^{3}, \cdots$ all generate different closed ideals in $A(\Gamma)$. The hypothesis of spectral synthesis asserts that any two closed ideals in $A(\Gamma)$ having the same "zero set" coincide-see below for definitions.)

Definitions. $G$ is an infinite discrete abelian group; $\Gamma$ is its dual, which is compact and not discrete. For $f \in L^{1}(G)$ the Fourier transform $\hat{f}$ is defined by $\hat{f}(\gamma)=\sum_{G} f(p)(-p, \gamma) d p$. For $f \in L^{1}(\Gamma)$ we set $\hat{f}(p)=\int_{\mathrm{r}} f(\gamma)(p, \gamma) d \gamma$, so that the Fourier inversion theorem holds: $f^{\wedge}=f . A(\Gamma)$ denotes the algebra of Fourier transforms $\hat{f}(\gamma), f \in L^{1}(G)$. It is endowed with the norm, $\|\hat{f}\| \equiv \sum|f(p)|$, so that $A(\Gamma)$ is just an isomorphic and isometric copy of $L^{1}(G)$. The zero set of an ideal $I \subseteq A(\Gamma)$ is the set of points $\gamma_{0} \in \Gamma$ such that $f\left(\gamma_{0}\right)=0$ for all $f \in I$. For $g \in A(\Gamma), \eta(g)$ denotes the $L^{\infty}$ norm of the sequence $\hat{g}: \eta(g) \equiv \sup |\hat{g}(p)|$, $p \in G$.

Malliavin showed (cf. [3], [4], [5]) that spectral synthesis fails in $L^{1}(G)$ provided there exists a real valued function $f \in A(\Gamma)$ such that, for $-\infty<u<\infty$

$$
\eta\left(e^{i u f}\right)=O\left(|u|^{-n}\right), \quad \text { all } n \geqq 0 .
$$

THEOREM. There exists a real valued function $f \in A(\Gamma)$ which satisfies (A).

Proof. To simplify the discussion, we will assume that $G$ is the group of integers ( $\Gamma$ is the circle group). The modifications needed for dealing with the general case are similar to those in Rudin [5,

${ }_{1}^{1}$ This work was partially supported by N.S.F. Grant GP4033. 
pp. 177-181]. Actually (A) can be improved upon; in fact, for any $s>1$, there exists a real valued function $f \in A(\Gamma)$ such that

$$
\eta\left(e^{i u f}\right)=O\left[\exp \left(-\delta|u|^{1 / s}\right)\right] \quad(\delta>0) .
$$

This function has the form

$$
f(\gamma)=\sum_{k=1}^{\infty}\left(1 / k^{s}\right) \cos p_{k} \gamma,
$$

where $p_{1}<p_{2}<\ldots$ is a "very rapidly" increasing sequence of integers. For the sake of definiteness, let us take $s=2$.

The proof of (B) is based on certain properties of the norm $\eta$. These are given in (a), (b), (c), Lemma 1 and Lemma 2.

REMARKs. Although $\eta$ is obviously a norm in the linear sense, it is not multiplicative (cf. Lemma 1). Proposition (c) depends on the fact that $G$ is the group of integers, and Lemmas 1 and 2 would have to be modified slightly for the case of an arbitrary $G$. The proofs of (a)-(c) are trivial.

(a) If $h \in A(\Gamma),|h| \leqq 1$, then $\eta(h)<1$ unless the Fourier series expansion of $h(\gamma)$ contains exactly one term.

(b) $\eta$ is continuous in terms of the topology on $A(\Gamma)$ (since $\eta($ ) $\leqq\|\|)$.

(c) For all integers $k \neq 0, \eta[g(k \gamma)]$ is independent of $k(g \in A(\Gamma))$.

Lemma 1. Let $g_{\alpha}$ and $h_{\alpha}$ be nonzero elements of $A(\Gamma)$ which depend continuously on a real parameter $\alpha$. Then for any $M>0$ and any $\epsilon>0$, there exists a positive integer $k$ such that

$$
\eta\left[g_{\alpha}(\gamma) \cdot h_{\alpha}(k \gamma)\right]<(1+\epsilon) \cdot \eta\left(g_{\alpha}\right) \cdot \eta\left(h_{\alpha}\right) \quad \text { for all } \alpha \in[-M, M] .
$$

SKETCH OF PROOF. The idea is that, if $k$ becomes very large, the nonzero Fourier coefficients of $h(k \gamma)$ will be very far apart. But by definition of $A(\Gamma)$, the Fourier series for $g_{\alpha}(\gamma)$ is absolutely convergent. Moreover, since $g_{\alpha}$ varies continuously in $A(\Gamma)$, the convergence can be shown to be uniform in $\alpha, \alpha \in[-M, M]$. Then (2) follows from the standard convolution formula for the Fourier coefficients of a product.

Lemma 2. For any interval $[a, b]$ not containing 0 , there exists a constant $\rho<1$ such that $\eta[\exp (i \alpha \cos k \gamma)] \leqq \rho$ for all $k \neq 0$ and all $\alpha \in[a, b]$.

Proof. Simply apply (a), (b), and (c) (a continuous function on a compact set assumes a maximum value).

Now to prove (B). Since $f(\gamma)=\sum_{k=1}^{\infty}\left(1 / k^{2}\right) \cos p_{k} \gamma$, it follows that $\exp (i u f(\gamma))=\prod_{k=1}^{\infty} b_{k}(\gamma, u)$ where

$$
b_{k}(\gamma, u) \equiv \exp \left[\left(i u / k^{2}\right) \cos p_{k} \gamma\right] .
$$


Define $c_{n}(\gamma) \equiv \prod_{k=1}^{n} b_{k}(\gamma)$. Choose $\left\{\epsilon_{k}\right\}, \epsilon_{k}>0$, so that $\Pi\left(1+\epsilon_{k}\right)<2$. Then from Lemma 1 , for a suitable choice of $\left\{p_{n}\right\}$ (defined by induction of course)

$$
\eta\left(c_{n+1}\right)<\left(1+\epsilon_{n}\right) \eta\left(c_{n}\right) \eta\left(b_{n+1}\right) \text { for }|u| \leqq n^{2} .
$$

(Note that the range of admissible values of $u$ increases with each $n$.) Thus, since $\eta$ is continuous on $A(\Gamma)$

$$
\eta\left(\prod_{k=1}^{\infty} b_{k}\right)<2 \eta\left(c_{n}\right) \prod_{k>n} \eta\left(b_{k}\right) \text { for }|u| \leqq n^{2} .
$$

Now break the interval $[1, \infty)$ and the sequence $\{1,2, \cdots\}$ into sections $4^{n-1} \leqq|u| \leqq 4^{n}, 2^{n}<k \leqq 2^{n+1}$. By Lemma 2 there exists a $\rho<1$ such that for any $u, k$ with $1 / 16 \leqq\left|u / k^{2}\right| \leqq 1, \eta\left(b_{k}\right) \leqq \rho$ (cf. (3)). Hence (5) implies

$$
\eta\left(\prod_{k=1}^{\infty} b_{k}\right)=O\left[\rho^{|u|^{1 / 2}}\right] \quad(\rho<1) .
$$

Proof. Consider $|u|$ in the interval $4^{n-1} \leqq|u| \leqq 4^{n}$; now replace $n$ by $2^{n}$ in (5), and look only at the terms $\eta\left(b_{k}\right)$ with $2^{n}<k \leqq 2^{n+1}\left(\eta\left(b_{i}\right)\right.$, $\eta\left(c_{i}\right) \leqq 1$ for all $i$, because $\left.\left|b_{i}\right| \equiv\left|c_{i}\right| \equiv 1\right)$.

Finally, since $\prod_{k=1}^{\infty} b_{k}=\exp (i u f),\left(B^{\prime}\right)$ is equivalent to (B) (with $s=2$ ).

Added in proof. The author has recently learned of another simplified proof of "non spectral synthesis" due to Kahane and Katznelson (Israel J. (1963); see also Stanford Univ. Seminar Notes by Katznelson, 1965). Their construction requires a little more analytic preparation than that given here; but it also proves more-e.g. that the function $f$ described above can be chosen to be Hölder continuous.

\section{REFERENCES}

1. J. P. Kahane, Sur un theorème de Paul Malliavin, C. R. Acad. Sci. Paris 248 (1959), 2943-2944.

2. J. P. Kahane and R. Salem, Ensembles parfaits et séries trigonometriques, Hermann, Paris, 1963.

3. P. Malliavin, Impossibilitê de la synthèse spectrale sur les groupes abéliens non compacts, Publ. Math. Inst. Hautes Etudes Sci. 1959, 61-68. 81-83.

4. W. Rudin, Closed ideals in group algebras, Bull. Amer. Math. Soc. 66 (1960),

5. - Fourier analysis on groups, Interscience, New York, 1962.

6. N. T. Varopoulos, Sur les ensembles parfaits et les séries trigonométriques. I, C. R. Acad. Sci. Paris 260 (1965), 4668-4670.

7. - Sur les ensembles parfaits et les séries trigonometriques. II, C. R. Acad. Sci. Paris 260 (1965), no. 21.

UNIVERSITY OF MinNeSOTA 